\title{
Correction to: Disturbed Gastrointestinal Contractility in a Polycystic Ovary Syndrome Rat Model
}

\author{
Kai-Lee Wang ${ }^{1,2}$ (D) Shih-Min Hsia ${ }^{3}$ Paulus S. Wang ${ }^{2,4,5,6} \cdot$ Po-Han Lin ${ }^{3}$
}

Published online: 20 April 2020

○) Springer Science+Business Media, LLC, part of Springer Nature 2020

\section{Correction to: Digestive Diseases and Sciences https://doi.org/10.1007/s10620-019-06001-x}

The original version of the article unfortunately contained an error in the legend of Figure 5B. Corrected version of Figure 5 is given below.
Publisher's Note Springer Nature remains neutral with regard to jurisdictional claims in published maps and institutional affiliations.

The original article can be found online at https://doi.org/10.1007/ s10620-019-06001-x.

Kai-Lee Wang

d49505002@gm.ym.edu.tw; kellywang@tmu.edu.tw

1 Department of Nursing, Ching Kuo Institute of Management and Health, No. 336, Fuxsin Rd., Zhongshan Dist., Keelung City 20301, Taiwan, Republic of China

2 Department of Physiology, School of Medicine, National Yang-Ming University, Taipei, Taiwan, Republic of China

3 School of Nutrition and Health Sciences, Taipei Medical University, Taipei 11031, Taiwan, Republic of China

4 Department of Medical Research and Education, Taipei Veterans General Hospital, Taipei, Taiwan, Republic of China

5 PhD Program of Aging, College of Medicine, China Medical University, Taichung, Taiwan, Republic of China

6 Department of Biotechnology, Asia University, Taichung, Taiwan, Republic of China 
Fig. 5 Effect of PCOS on stomach $\mathrm{Ca}^{2+}$ mobilization and muscle constriction. The stomach was cultured in calcium-free Krebs solution to eliminate extracellular calcium. Afterward, a high concentration of carbachol $(10 \mu \mathrm{M})$ was used to evaluate the ability of intracellular calcium release. Thereafter, a $\mathrm{CaCl}_{2}$ solution was added cumulatively into the organ bath to elicit a sustained stomach muscle contraction. The figure is expressed as final concentration of calcium. Muscle contractility (a) and the area under the curve (b) were shown. Four to five different stomach segments prepared from different rats were used in the experiment. The experiment was performed twice, and similar results were obtained. Each value is presented as mean \pm SEM $(n=4-5)$. $* P<0.05 ; * * P<0.01$ versus the vehicle group
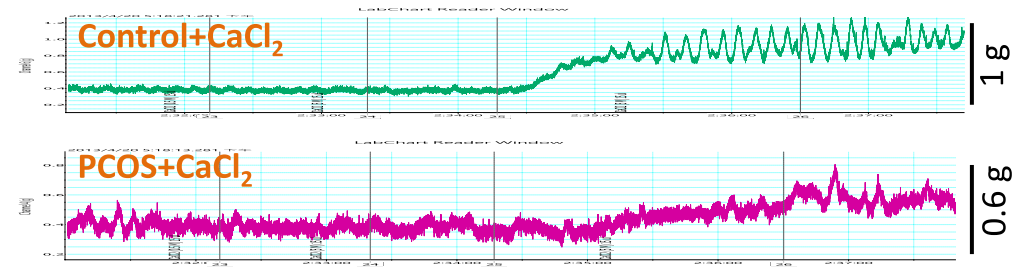

B

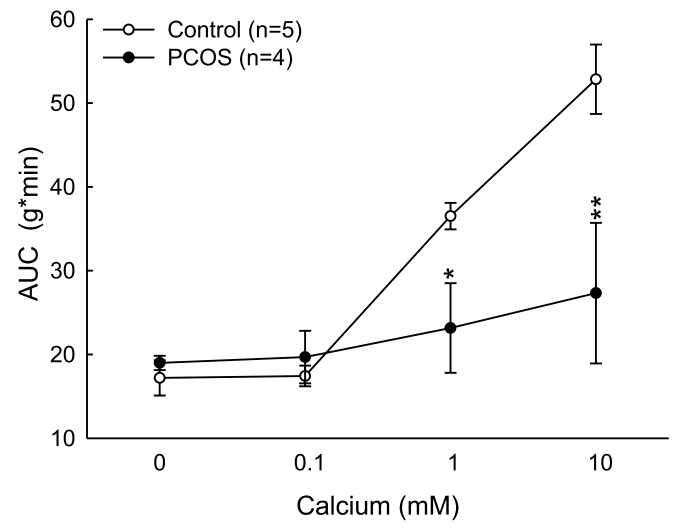

